\title{
Funcionalidad familiar del paciente con VIH-SIDA en una Unidad de Medicina Familiar de León, Guanajuato
}

\author{
Family function of HIV-AIDS patient in a Family \\ Practice Center in León, Guanajuato \\ Danica Caldera-Guzmán, * María del Pilar Pacheco-Zavala
}

\begin{abstract}
RESUMEN. Introducción: La prevalencia de VIH/SIDA en México es relativamente estable. En el caso del estado de Guanajuato, en 2016, ésta presentó una proporción de 2\%. Esta patología transita por múltiples estadios de infección, durante los cuales se requieren diferentes grados de apoyo familiar, lo que, con frecuencia, implica la reestructura de las relaciones familiares. Objetivo: Conocer la funcionalidad familiar de los pacientes con VIH-SIDA en la Unidad de Medicina Familiar (UMF) Núm. 53 en León, Guanajuato. Material y métodos: Estudio transversal simple, realizado en la UMF Núm. 53 en León, Guanajuato. Se incluyeron a 71 pacientes de la consulta externa, incluidos de ambos turnos, durante el periodo de agosto a diciembre del 2018. Para determinar la funcionalidad familiar del paciente portador de VIH-SIDA, se utilizó el cuestionario Apgar. Resultados: De los 71 pacientes, sólo fueron localizables 65, con una mayor prevalencia de solteros (43\%), siendo principalmente del género masculino (71\%) y escolaridad preparatoria; $64 \%$ presentó algún grado de disfunción familiar. Conclusiones: Se encontró que hay un predominio de disfunción familiar, siendo de mayor relevancia la falta de apoyo con relación a la adaptación, la participación y el crecimiento, así como el ser soltero, lo cual se traduce en una menor red de apoyo. Estos factores favorecen una menor adherencia al tratamiento y una mayor prevalencia de depresión y ansiedad.
\end{abstract}

Palabras clave: VIH-SIDA, funcionalidad familiar, Apgar.

ABSTRACT. Introduction: The prevalence of HIV/AIDS in Mexico is relatively stable; Guanajuato has a proportion of $2 \%$ in 2016. This pathology passes through multiple stages of infection, during which they require different degrees of family support, which often implies restructuring. of family relationships. Objective: Know the family function of patients with HIV-AIDS at UMF No. 53 in León, Guanajuato. Material and methods: Simple cross-sectional study, conducted at UMF No. 53 in León, Guanajuato. 71 patients were included in the outpatient consultation of both shifts during the period from august to December 2018. To determine the family function of the patient with HIV-AIDS, the Apgar questionnaire was used. Results: Of the 71 patients, only 65 were found, with a higher prevalence of singles (43\%), male gender (71\%) and preparatory schooling,

* Residente de tercer año.

‡ Adscrito.

Especialidad en Medicina Familiar. Unidad de Medicina Familiar (UMF) Núm. 53, Instituto Mexicano del Seguro Social (IMSS). León, Guanajuato, México.

Recibido: 30 de Octubre de 2019.

Aceptado: 31 de Mayo de 2020.
Correspondencia:

Dra. Danica Caldera Guzmán

Circuito el Mezquital Núm. 101,

Fraccionamiento La Mezquitera,

Col. Calzada los Mezquites, C.P. 76912 ,

Aguascalientes, Aguascalientes, México.

E-mail: dannie.cg@gmail.com

Conflicto de intereses: Todos los autores declaran que no existe ningún conflicto de intereses con respecto a la publicación de este artículo. 
$64 \%$ had some degree of family dysfunction. Conclusions: It was found that there is a predominance of family dysfunction, the lack of support in relation to adaptation, participation and growth being more relevant, as well as being single, which translates into a smaller support network favoring a lower treatment adherence and a higher prevalence of depression and anxiety.

Keywords: HIV-AIDS, family function, Apgar.

\section{INTRODUCCIÓN}

La infección por virus de inmunodeficiencia humana (VIH) es causada por un virus tipo 1 o 2 , el cual pertenece al género de los Lentivirus y, clínicamente, se caracteriza por una infección asintomática durante un periodo variable de hasta alrededor ocho años, debido al equilibrio entre la replicación viral y la respuesta inmunológica del paciente. Posteriormente, se rompe este equilibrio y se aumenta la carga viral, lo que deteriora la función inmune y permite la aparición de diversas infecciones clásicas oportunistas y tumores, con lo que se llega a la etapa de SIDA. ${ }^{1}$

Según el Registro de Nacional de Casos de SIDA, al 15 noviembre de 2016, los casos notificados de VIH-SIDA de los años 1983 a 2016 son 186,665 habitantes. De éstos, se encuentran vivos 135,515 y según el estado de evolución registrado se distribuyen en SIDA: 72,599 y VIH: 62,916. De acuerdo con el CENSIDA, los estados con la mayor tasa (tasa por 100,00 habitantes) de casos nuevos diagnosticados de VIH y de SIDA en 2016 son: para SIDA, Campeche con $22.1 \%$, Guerrero con 9.8\%, Morelos con $7.9 \%$, Colima con $7.7 \%$, Tlaxcala con $7.7 \%$, y para VIH, lo son Campeche con $17.4 \%$, Yucatán con $13.7 \%$, Quintana Roo con 7.8\%, Tabasco con $7.6 \%$, Oaxaca con $6.7 \% .^{2}$

El estado de Guanajuato tiene una epidemia relativamente baja y estable que, de acuerdo con las estadísticas de CENSIDA, de los años 1983 a 2016 se ha obtenido un total de 3,644 pacientes; de éstos, 3,054 son hombres y $590 \mathrm{mu}$ jeres, teniendo tasa de proporción total del $2 \%{ }^{2}$

En la Unidad de Medicina Familiar (UMF) Núm. 53 se cuenta con un censo total de 332 pacientes durante el presente año, de los cuales 126 son portadores de SIDA y 106 seropositivos. Cabe mencionar que, de este total, sólo 39 son portadores de SIDA y 32 portadores de seropositivo acuden a control a la unidad.

Una familia funcional es capaz de cumplir con las tareas que le están encomendadas de acuerdo con la etapa del ciclo vital en la que se encuentre y en relación con las demandas que percibe desde su ambiente externo; es una mezcla de sentimientos, comportamientos y expectativas entre los miembros de la familia, que le permiten a cada uno de ellos desarrollarse como individuo, y les infunde, además, el sentimiento de no estar aislado y poder contar con el apoyo de los demás. ${ }^{3}$

La relación entre apoyo social, depresión, afrontamiento y salud parece ser especialmente importante para las personas que viven con el VIH. Zich y Temoshok demostraron que los pacientes portadores de VIH-SIDA con falta de apoyo social y familiar presentaron mayores de episodios de recaídas y una mayor exacerbación de síntomas físicos. ${ }^{4}$

En el caso de los pacientes infectados por VIH, la depresión suele ser de dos a cuatro veces más frecuente que en la población general, considerándose como una de las comorbilidades psiquiátricas más frecuentes, y la de mayor incidencia tras el diagnóstico de la infección. ${ }^{5-8}$

Se ha reportado que la depresión en sí puede afectar negativamente la evolución de la infección por VIH, pues un peor estado inmunológico se expresa en un menor recuento de linfocitos $\mathrm{T}$ CD4+, mayor carga viral, progresión de la enfermedad y mayor mortalidad. ${ }^{8,9}$

Los instrumentos de evaluación de la familia nos permiten identificar en qué medida se cumplen sus funciones básicas y cuál es el rol que puede estar jugando la familia en el proceso salud-enfermedad. El Dr. Gabriel Smilkstein de la Universidad de Washington, Seattle, en 1978, creó el Apgar familiar como una respuesta a la necesidad de evaluar la función de la fa- 
milia. Éste se diseñó para el uso potencial de personas en distintos estratos socioeconómicos y en diversos contextos socioculturales; además, es capaz de proporcionar datos que indiquen la integridad de componentes importantes de la función familiar. Sus parámetros a evaluar son la adaptación, participación o cooperación, ganancia o crecimiento, afecto y recursos. ${ }^{6}$

Dentro de la medicina familiar, nuestro estudio no es solamente el enfermo, sino todo su entorno, pero sobre todo su familia, por lo que no debemos olvidar cómo ésta se ve afectada. En el caso de los pacientes con VIH-SIDA, hay poca evidencia sobre el apoyo familiar, por lo que se pretende explorar el apoyo social y familiar en pacientes portadores en cualquier estadio, indagando el grado de función familiar que acarrearía en su entorno durante el tiempo de evolución de su enfermedad a partir de la raíz del diagnóstico, de tal manera que nos permitirá abrir espacios de conciencia mutua entre el enfermo y su entorno familiar con el fin de lograr una acción colectiva contra el virus y una mejor convivencia con las personas afectadas por el mismo, además de que se reforzaría la importancia del apoyo familiar durante la trayectoria de la enfermedad, la generación de solidaridad y compromiso frente al desafío que plantea el SIDA. ${ }^{8,10-12}$

\section{MATERIAL Y MÉTODOS}

Se realizó un estudio observacional, prospectivo, transversal y descriptivo durante los me-

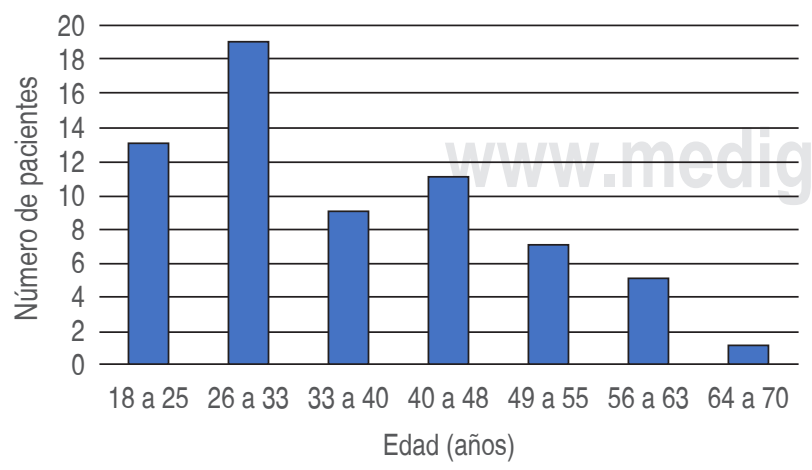

Figura 1: Distribución del número de pacientes por intervalo de edad.

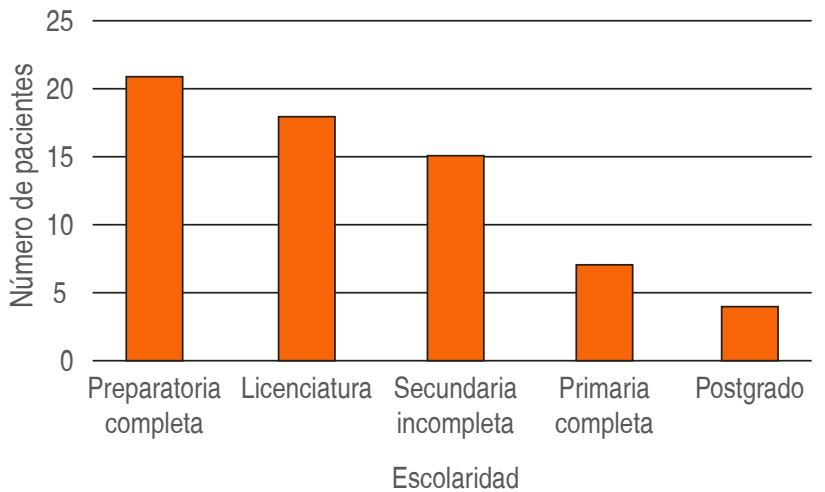

Figura 2: Número de pacientes por nivel máximo de escolaridad.

ses de agosto a diciembre de 2008, en el cual se incluyeron a todos los pacientes portadores de VIH-SIDA adscritos de ambos turnos a la consulta externa de la Unidad de Medicina Familiar Núm. 53. A ellos se les explicó el objetivo de la investigación y se les solicitó que firmaran un consentimiento informado; posteriormente, se aplicó una encuesta para evaluar datos generales y el test de Apgar familiar.

El manejo sistematizado de los datos se llevó a cabo con la utilización del programa SPSS versión 10, con el cual, además, se realizó la determinación del manejo estadístico de cada uno de los indicadores identificados (se utilizaron frecuencias simples y proporciones). Asimismo, se elaboraron cuadros y figuras representativas de los datos obtenidos.

\section{RESULTADOS}

De los 71 pacientes adscritos a la UMF Núm. 53 portadores de VIH SIDA de los turnos matutinos y vespertinos, sólo fueron localizables 65 . De estos 65 pacientes, 46 fueron hombres y 19 mujeres, siendo el género masculino el de mayor prevalencia, con un promedio de edad de 35.8 años ( \pm 12.25$)$,

La prevalencia de portadores seropositivos y SIDA, con relación al género, fue de 39 y $34 \%$ en los hombres respectivamente; en las mujeres, $20 \%$ y $7 \%$ respectivamente. $\mathrm{Al}$ determinar el intervalo de edad en el cual se encontraban los pacientes, se observó que el más frecuente fue de 26 a 33 años con 29\%; el de menor frecuen- 
cia fue de 64 a 70 años con 1\% (Figura 1). Al analizar la escolaridad, el mayor porcentaje fue de preparatoria completa con $32 \%$, seguido de licenciatura con $28 \%$ (Figura 2). Con relación al estado civil, se observó un mayor porcentaje de solteros y en unión libre con $81 \%$ en total (Figura 3). Al analizar los resultados de la aplicación del Apgar familiar, se encontró que 64\% presentó disfunción familiar en algún grado. Asimismo, 36\% tiene una buena funcionalidad familiar (Figura 4). Con relación a las respuestas obtenidas en cada uno de los ítems del Apgar familiar, resalta que $36 \%$ de los pacientes no solicita ayuda a la familia cuando se enfrentan a un problema y $31 \%$ no se encuentra satisfecho con la forma en que la familia los apoya.

Finalmente, no se observaron inconvenientes en relación a cómo la familia acepta y apoya sus deseos de emprender nuevas actividades, así como la muestra de afecto que reciben por parte de los miembros de la familia y cómo se comparten los espacios, el tiempo y el dinero (Tabla 1).

\section{DISCUSIÓN}

Las características sociodemográficas del grupo de pacientes con infección por VIH-SIDA en este estudio sugieren una evolución y particularidades propias de la enfermedad, similar a lo encontrado en 2011 por Cambambia-Jiménez y colaboradores en una Unidad de Primer Nivel

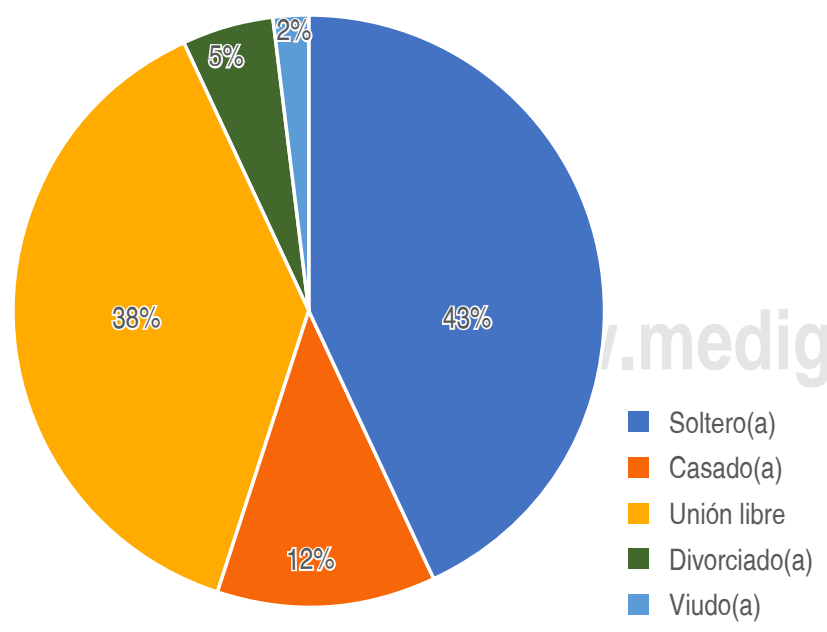

Figura 3: Porcentaje de prevalencia de estado civil.

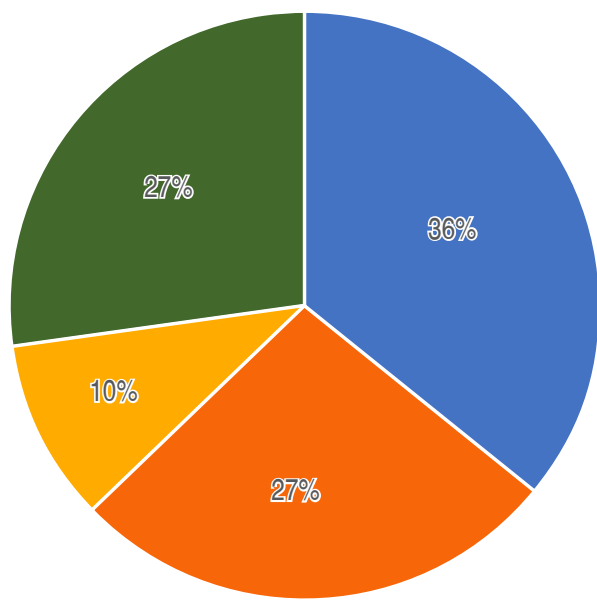

Buena funcionalidad familiar

Disfunción familiar moderada

Disfunción familiar leve

Disfunción familiar severa

Figura 4: Funcionalidad familiar.

en Xalapa, México. Ellos reportaron una mayor prevalencia del género masculino (74\%), así como similitudes en la escolaridad, con un predominio de licenciatura en $41.5 \%$ y en el estado civil, encontrando un mayor porcentaje de solteros (36.9\%). Además, los hallazgos encontrados en este estudio con relación a la funcionalidad familiar fueron similares, con predominio de las familias disfuncionales. ${ }^{7}$

En lo que concierne a la evaluación de cada uno de los ítems, se encontró que 36\% de los pacientes no se siente con la confianza de solicitar ayuda a los integrantes de su familia ante la presencia de alguna problemática a la que se enfrentan; $31 \%$ no se encuentra satisfechos con el apoyo de la familia para emprender nuevas actividades sea de índole laboral o personal, y $25 \%$ destacó que no se comparten mensajes verbales y/o afectivos por parte de los demás miembros de familia. Contrastando los resultados obtenidos, se pudo encontrar similitud a lo reportado en un estudio en 2014 en Nigeria, donde se evaluó la asociación de la funcionalidad familiar con los síntomas depresivos en el paciente portador de VIH/SIDA. ${ }^{8}$

En México, se menciona en forma circunstancial el tema de familia con relación al VIH/ SIDA; al respecto, existen escasas investigaciones que mencionen las estrategias a las que re- 
curren las familias de personas infectadas por VIH para enfrentar y manejar la enfermedad, debido a que suele asumirse que los enfermos cuentan con una red de apoyo familiar. ${ }^{9}$

El estigma y la discriminación son realidades del día a día para las personas que viven con VIH, por lo que la tendencia más común es ocultar la enfermedad. El dilema de hablar acerca del VIH obliga a las personas al aislamiento, evitando el rechazo de los miembros de su familia. ${ }^{8-10}$

En el entorno social, la mayoría de las personas infectadas con VIH/SIDA optan por vivir en incógnito, esto debido a que la población desconoce la enfermedad y es muy fácil para la familia, amigos, vecinos mantener perjuicios y tabúes, los cuales orillan al paciente a enfrentar este problema de manera individual. Por esto es por lo que el conocimiento de la enfermedad y la comunicación constituyen una necesidad básica para todos los miembros de la familia. ${ }^{8,10}$

El desconocimiento sobre la enfermedad crea barreas que dificultan la unión de la familia; la convivencia directa, afectiva y vinculante redefine el VIH como una actitud empática no estigmatizadora. La esperanza, el acompaña- miento familiar y la información permiten a los pacientes con VIH una percepción diferente de su enfermedad.

Una de las funciones esenciales de la familia es el apoyo social. Sabemos que éste es uno de los factores de mayor importancia para la vida del paciente con VIH. Es importante que el paciente cuente con distintas redes, abarcando desde la comunicación empática y bilateral por parte de la familia, compañeros de trabajo, parejas, vecinos e instituciones de salud, pues con ello se podrá brindar aliento ante una evolución tórpida y durante los procesos infecciosos agregados que implica la evolución natural del VIH.

Es destacable que la presencia de la enfermedad obligue a un momento de transición y de redefinición del concepto de la familia, por lo que se debe enfatizar en que ésta siga realizando, aunque sea parcialmente, las tareas del momento del ciclo vital familiar en que se encuentra, evitando la detención del paciente, enfrentando las tareas nuevas y los roles modificados ante la presencia del VIH. Las familias funcionales brindan una afirmación positiva a todos sus miembros, permitiendo que cada uno

Tabla 1: Ítems evaluados en Apgar familiar.

\begin{tabular}{|c|c|c|c|c|c|}
\hline \multirow[b]{2}{*}{ Apgar } & \multicolumn{5}{|c|}{ Número de sujetos (\%) } \\
\hline & Siempre & $\begin{array}{l}\text { Casi } \\
\text { siempre }\end{array}$ & $\begin{array}{l}\text { Algunas } \\
\text { veces }\end{array}$ & $\begin{array}{c}\text { Casi } \\
\text { nunca }\end{array}$ & Nunca \\
\hline $\begin{array}{l}\text { Adaptación } \\
\text { Me satisface la ayuda que recibo de mi familia cuan- } \\
\text { do tengo algún problema y/o necesidad }\end{array}$ & $17(26)$ & $23(36)$ & $15(23)$ & $6(9)$ & $4(6)$ \\
\hline $\begin{array}{l}\text { Participación o cooperación } \\
\text { Me satisface la participación que mi familia brinda y } \\
\text { permite }\end{array}$ & $19(29)$ & $20(31)$ & $13(20)$ & $5(8)$ & $8(12)$ \\
\hline $\begin{array}{l}\text { Ganancia o crecimiento } \\
\text { Me satisface cómo mi familia acepta y apoya mis } \\
\text { deseos de emprender nuevas actividades }\end{array}$ & $20(31)$ & $20(31)$ & $10(15)$ & $8(12)$ & $7(11)$ \\
\hline $\begin{array}{l}\text { Afecto } \\
\text { Me satisface cómo mi familia expresa afectos y res- } \\
\text { ponde a mis emociones, como rabia, tristeza o amor }\end{array}$ & $22(34)$ & $16(25)$ & $15(23)$ & $9(14)$ & $3(4)$ \\
\hline $\begin{array}{l}\text { Recursos } \\
\text { Me satisface cómo compartimos en familia: el tiempo } \\
\text { para estar juntos, los espacios en la casa y el dinero }\end{array}$ & $21(32)$ & $19(29)$ & $11(17)$ & $9(13)$ & $5(9)$ \\
\hline
\end{tabular}


se sienta querido y valorado. Si la familia tiene las herramientas y el apoyo necesario para hacer frente a la situación y brinda apoyo al paciente, surgirán los cambios necesarios para una mejor adaptación, tanto para el enfermo como para los integrantes de la familia. ${ }^{8-11}$

La información que se reporta nos hace notar que buena parte de la problemática que rodea al paciente con VIH/SIDA sucede en el ámbito familiar. El médico familiar es el único especialista que maneja de forma integral a un paciente involucrado en las distintas problemáticas de salud que se presentan a lo largo de la vida de un individuo, por lo que es relevante entender que el VIH/SIDA es una enfermedad como cualquier otra, que es tratable y que, por su endemia, posee una complejidad, la cual va más allá de una crisis de salud, tomando en cuenta que no se intenta enfrentar solamente una alteración biológica, sino que se acompaña de temores psicológicos, reacciones sociales de pánico, ignorancia, prejuicios y discriminación, incluso por parte de la familia. ${ }^{12}$ Como parte de esta atención integral, se debe fomentar la participación protagónica de todos los miembros de la familia al individuo, la convivencia y la ejecución de acciones que tengan un impacto positivo en las personas que viven con VIH.

Una limitación de este estudio fue que en la evaluación de la funcionalidad familiar sólo se tomó en cuenta la percepción del paciente, por lo que en estudios posteriores se podría implementar la aplicación del instrumento a todos los miembros de la familia; otro punto a resaltar es que, debido al desconocimiento de la enfermedad por todos los miembros de la familia, la impresión de la funcionalidad familiar del paciente pudiera estar sesgada.

\section{CONCLUSIONES}

Existe un predominio de algún grado de disfunción familiar en los pacientes con VIH/SIDA, siendo de mayor relevancia la falta de apoyo con relación a la adaptación, la participación y el crecimiento, así como el ser soltero, lo cual se traduce en una menor red de apoyo para los pacientes. Estos aspectos favorecen una menor adherencia al tratamiento y una mayor prevalecía de depresión y ansiedad.

\section{BIBLIOGRAFÍA}

1. Benett C. Tratado de medicina interna. $20^{\mathrm{a}}$. ed. México: Interamericana; 2009.

2. Secretaría de Salud. Vigilancia epidemiológica de casos VIH/SIDA en México. Registro Nacional de Casos de SIDA. Actualización al 15 de noviembre de 2016. 2016.

3. Mendoza-Solís LA, Soler-Huerta E, Sainz-Vázquez L et al. Análisis de la dinámica y funcionalidad familiar en atención primaria. Arch Med Fam. 2006; 8 (1): 27-32.

4. Ji G, Li L, Lin C, Sun S. The impact of HIV/AIDS on families and children--a study in China. AIDS. 2007; 21 Suppl 8 (Suppl 8): S157-S161.

5. Gonzalez JS, Batchelder AW, Psaros C, Safren SA. Depression and HIV/AIDS treatment nonadherence: a review and meta-analysis. J Acquir Immune Defic Syndr. 2011; 58 (2): 181-187.

6. Román-López CA, Angulo-Valenzuela RA, FigueroaHernández G et al. El médico familiar y sus herramientas. Rev Med MD. 2015; 6 (4): 294-299.

7. Cambambia-Jiménez GA, Sainz-Vázquez L, GalindoSainz J et al. Perfil de las familias de pacientes con VIH/
SIDA de una Unidad de Medicina Familiar de Xalapa, México. Arch Med Fam. 2010; 12 (3): 77-82.

8. Shittu RO, Issa BA, Olanrewaju AT, Mahmoud AO, Odeigah LO. Family dysfunction and depressive symptoms among people living with HIV/AIDS, in Nigeria, West Africa. IJHRS. 2014; 3 (1): 7-18.

9. Castro R, Eroza E, Orozco E, Manca M, Hernández J. Estrategias de manejo en torno al VIH/SIDA. Salud Pública Mex. 1997; 39 (1): 32-43.

10. Pretorius J, Greeff M, Freeks F, Kruger A. A HIV stigma reduction intervention for people living with HIV and their families. Health SA Gesonheid. 2016; 21 (26): 187-195.

11. Galindo SJ, Ortega RM. Prevalencia de depresión en pacientes con VIH/SIDA en el Hospital General de Zona \# 11 del Instituto Mexicano del Seguro Social (IMSS), Lic. Ignacio Díaz Téllez. Enf Infec Microbiol. 2010; 30 (4): 129-133.

12. Briongos Figuero LS, Bachiller Luque P, Eiros Bouza JM, Palacios Martín T. Papel del médico de familia en el manejo de la infección por VIH. An Med Interna (Madrid). 2007; 24 (8): 399-403. 